\title{
Subclinical concentrations of sevoflurane reduce oxidative stress but do not prevent hippocampal apoptosis
}

\author{
ZHI-BIN ZHOU ${ }^{1}$, XIAO-YU YANG ${ }^{1}$, YING TANG ${ }^{2}$, XUE ZHOU ${ }^{1}$, LI-HUA ZHOU ${ }^{2}$ and XIA FENG ${ }^{1}$ \\ ${ }^{1}$ Department of Anesthesiology, The First Affiliated Hospital of Sun Yat-sen University; \\ ${ }^{2}$ Department of Anatomy, Zhong Shan Medical College, Sun Yat-sen University, Guangzhou, Guangdong 510080, P.R. China
}

Received February 1, 2015; Accepted December 9, 2015

DOI: $10.3892 / \mathrm{mmr} .2016 .5336$

\begin{abstract}
Sevoflurane is generally considered a pro-apoptotic agent in the neonatal brain. However, recent studies have suggested that low levels of sevoflurane anesthesia may be neuroprotective and have a memory enhancing effect. The present study aimed to investigate whether sevoflurane exerts a neuroprotective effect at subclinical concentrations, with regard to oxidative state. In the current study, postnatal day 7 (P7) Sprague-Dawley rats were continuously exposed to 0.3 , 1.3 , or $2.3 \%$ sevoflurane for $6 \mathrm{~h}$. ELISA was used to quantify the levels of superoxide dismutase, glutathione peroxidase (GSH-px) and malondialdehyde (MDA) in the plasma and the hippocampus. Terminal deoxynucleotidyl-transferase dUTP nick-end labeling staining was used to observe hippocampal neuronal apoptosis. Altered object exploration tests for recognition memory were employed to investigate long-term behavioral effects at postnatal day 28 . The results demonstrated that a single $6 \mathrm{~h}$ exposure to a subclinical concentration (1.3\%) of sevoflurane at P7 reduces MDA and GPH-px production in rats. Sevoflurane induced hippocampal apoptosis in a dose-dependent manner and altered recognition memory testing indicated no differences among the groups. Although early exposure to a subclinical concentration of sevoflurane reduced oxidative stress, it did not prevent the process of sevoflurane-induced hippocampal apoptosis. These changes did not affect subsequent recognition memory in juvenile rats.
\end{abstract}

Correspondence to: Professor Xia Feng, Department of Anesthesiology, The First Affiliated Hospital of Sun Yat-sen University, 58 Zhongshan 2nd Road, Guangzhou, Guangdong 510080, P.R. China

E-mail: fengxia@mail.sysu.edu.cn

Abbreviations: SOD, superoxide dismutase; GSH-px, glutathione peroxidase; MDA, malondialdehyde; ELISA, enzyme linked immunosorbent assay

Key words: sevoflurane, oxidative, hippocampus, apoptosis, recognition

\section{Introduction}

Sevoflurane inhalation is a popular general anesthetic option for pediatric patients (1). It has non-hepatic, non-renal dependent elimination features and low solubility, thus providing faster induction and emergence qualities than the majority of other commonly used general anesthetics (2). However, results of recent animal research regarding the neural safety of sevoflurane are paradoxical. Sevoflurane is considered to be neuroprotective in certain preconditioning situations $(3,4)$, but it has also been observed to induce widespread neuronal apoptosis in the mouse brain at certain concentrations $(5,6)$.

Behavioral investigations have demonstrated that subanesthetic concentrations of volatile anesthetics may enhance learning and memory in mice (7). Learning and memory involve synaptic plasticity, which is exemplified by long-term potentiation of the excitatory postsynaptic potential (8). Tight-seal whole-cell recordings have demonstrated that sevoflurane at subanesthetic concentrations [0.05-0.07 minimum alveolar concentration (MAC)] increases excitatory synaptic transmission in the region I of hippocampus proper (CA1) area, however, at 0.36 MAC it inhibits this action (9).

These observations suggest a low level of sevoflurane anesthesia may enhance learning and memory, possibly associated with neuroprotective regulation in hippocampal apoptosis signaling pathways. As various agents that induce apoptosis are either oxidants or stimulators of cellular oxidative metabolism (10), sevoflurane may affect oxidative stress in the hippocampus. To investigate this hypothesis, systemic and hippocampal oxidative status, neuronal apoptosis and recognition memory were examined following sevoflurane exposure at three different concentrations: Subanesthetic (0.3\%, 0.1 MAC; LS) $(11,12)$, subclinical (1.3\%, 0.3 MAC; MS) (13), and the highest tolerated concentration (2.3\%,0.7 MAC; HS) (14). The highest tolerated concentration was based on previous experiments that confirmed a substantial apoptosis-inducing effect under an established sevoflurane exposure system $(14,15)$.

\section{Materials and methods}

Animals. Animal use in the present study was authorized by the Institutional Animal Care and Use Committee at Sun Yat-sen University (Guangzhou, China). All attempts were made to use a smaller number of animals and decrease their 
suffering. Male Sprague-Dawley (SD) rats were acquired from the Experimental Animal Center of Sun Yat-sen University. They were accommodated in a room with a 12-h light-dark cycle (light from 07:00 to 19:00), and the room temperature was maintained at $21 \pm 1^{\circ} \mathrm{C}$. Food and water were provided ad libitum. The rats were housed in the same room in different cages. Each cage contained a different litter. Rats of different group allocation were marked by ear punching.

Earlier studies described litter variability in the rate of apoptosis among neonate mice (16). Therefore, an equal number of control and experimental rats were taken from the same litters so that each experiment had its own group of littermate controls. Only male rats were used including 96 pups from 16 litters.

Sevoflurane exposure. SD rats of postnatal day 7 (P7; weight, 16-17 g) were randomly allocated into 4 groups: An air-treated control group and 0.3, 1.3 and 2.3\% sevoflurane (Abbott Laboratories, Abott Park, IL, USA) treatment groups, with 21 rats in each treatment group. Rats in the sevoflurane treatment groups were placed in a plastic chamber and exposed to $0.3,1.3$ or $2.3 \%$ sevoflurane for $6 \mathrm{~h}$ with air as a carrier at a gas flow of $21 / \mathrm{min}$. During sevoflurane exposure, the chamber was heated to $38^{\circ} \mathrm{C}$ using a warming device (NPS-A3; Midea Group Co. Ltd., Foshan, China). Sevoflurane, oxygen and carbon dioxide levels in the chamber were calibrated by a gas monitor (Datex-Ohmeda, Inc., Madison, WI, USA). After 6 h, sevoflurane delivery ceased, and the animals were exposed to air again. When these rats moved freely, they were placed back into the maternal cage. During anesthesia, the respiratory frequency and skin color of the rats were monitored by an investigator. If signs of apnea or hypoxia were observed, the rat was exposed to air immediately and excluded from the experiment. Rats in the control group were placed into the same container as those in the sevoflurane groups but exposed to air for $6 \mathrm{~h}$.

Arterial blood gas analysis. P7-P8 rats from the sevoflurane-treated groups and the air-treated group underwent arterial blood gas analysis according to a procedure described previously (17). Samples were obtained immediately prior to ( $0 \mathrm{~h}, \mathrm{n}=3$ in each subgroup) and following anesthesia ( $6 \mathrm{~h}, \mathrm{n}=3$ in each subgroup) from a total of 12 rats. Briefly, the rats underwent a quick arterial blood sampling from the left cardiac ventricle via trans-thoracic puncture, and the samples were transferred to heparinized glass capillary tubes (Tianhong Glass Co., Taixin, China). A single sample (100 $\mu$ l) was analyzed immediately following blood collection by a blood gas analyzer (GEM Premier 3000; Instrumentation Laboratory, Barcelona, Spain). The $\mathrm{pH}$, arterial carbon dioxide tension, arterial oxygen tension, and blood glucose levels of arterial blood were analyzed. At the time of blood sampling, the experiments were terminated when the rats were sacrificed by decapitation. The analysis of each sample was repeated independently a minimum of three times.

Enzyme-linked immunosorbent assay (ELISA). Plasma and hippocampal homogenate were collected at $6 \mathrm{~h}$ following sevoflurane exposure. For plasma collection, blood samples were obtained from the left ventricle and transferred into heparinized tubes. The samples were centrifuged for $30 \mathrm{~min}$ at $3,000 \mathrm{x} \mathrm{g}$ at $4^{\circ} \mathrm{C}$ within $30 \mathrm{~min}$ of collection. Supernatants of the blood samples were stored at $-80^{\circ} \mathrm{C}$ for later use in the plasma ELISA. For hippocampal homogenates, brain tissue was removed following rapid decapitation. Bilateral hippocampi were dissected in $0^{\circ} \mathrm{C}$ phosphate-buffered saline (PBS) on ice under an XTX-4A microscope (Xindiweiye Medical Instrument Co., Ltd., Wuhan, China). Hippocampal tissue was homogenized in $1 \mathrm{ml} / \mathrm{g}$ of $0^{\circ} \mathrm{C} \mathrm{PBS}$, and the homogenate was stored at $-80^{\circ} \mathrm{C}$ for later use in ELISA. ELISA assays were performed according to the protocols of the commercial kits for rat superoxide dismutase (SOD), malondialdehyde (MDA) and glutathione peroxidase (GSH-px; Kaysam Bio-Technology Co., Ltd, Guangzhou, China). The results were obtained by using a microplate reader (Thermo MK3, Thermo Fisher Scientific Inc., Waltham, MA, USA).

Histopathological examination. Rats in the three sevoflurane-treated groups and the air-treated control group were sacrificed $6 \mathrm{~h}$ after the 6-h exposure to sevoflurane/air for terminal deoxynucleotidyl-transferase dUTP nick end labeling (TUNEL) staining. The animals were anesthetized with a fatal dose of $10 \%$ chloral hydrate (Huakai Resin Co., Ltd., Jining, China) and perfused transcardially with normal saline until the liver and lungs were clear of blood, a fixative of $4 \%$ paraformaldehyde (Tange Biotechnology Co., Ltd., Shanghai, China) in $0.1 \mathrm{M}$ phosphate buffer ( $\mathrm{pH}$ 7.4) was then perfused. The perfusion continued for 15-25 min, the brains were subsequently detached and preserved in the same fixative overnight. Paraffin blocks of the brain tissue $(0.5 \mathrm{~mm}$ thick) included different levels of the hippocampus along the septotemporal axis and associated areas (18). Paraffin coronal sections of the hippocampus (5- $\mu \mathrm{m}$ thick) underwent TUNEL staining (Roche, Basel, Switzerland) and were stained with hematoxylin. The results were examined in detail under a light microscope (Nikon ECLIPSE 50i; Nikon Corporation, Tokyo, Japan) to observe the morphological changes of the pyramidal neurons in CA1 and region III of hippocampus proper. TUNEL-positive neuronal cell numbers in the bilateral CA1 of the hippocampal pyramidal cell layer ( 3 sections per animal, $\mathrm{n}=3$ for each group) were counted by two individuals blinded to the groupings (14). Cells were counted at magnification $x 400$ only when neuronal structures of appropriate size and shape were demonstrated clearly with a TUNEL-positive nucleus. Uncertain structures were examined under magnification $\times 1,000$ and were not counted if the identification remained unclear (19).

\section{Behavioral studies}

Object exploration tests for recognition memory. Following postnatal day 28, the rats underwent an object exploration test (Fig. 1). This was based on a partial modification of the recognition memory test protocol (20). It was performed in a white plastic cubical box $(60 \times 60 \times 60 \mathrm{~cm})$ and between each trial, the box was wiped with $40 \%$ ethanol. In the object-in-place test, the north wall of the box was painted black (Fig. 1E). Subjects underwent three habituation periods prior to the first test (object recognition test). During each habituation period, rats were brought individually into the box to stay for $10 \mathrm{~min}$. Each period was separated by a $24 \mathrm{~h}$ interval. The object recognition test 
Table I. Arterial blood gas analysis prior to and following sevoflurane exposure.

Arterial blood

\begin{tabular}{lcccccc}
\cline { 3 - 6 } Group & Time $(\mathrm{h})$ & $\mathrm{n}$ & $\mathrm{pH}$ & $\mathrm{PaCO}_{2}(\mathrm{kPa})$ & $\mathrm{PaO}_{2}(\mathrm{kPa})$ & $\mathrm{Glucose}\left(\mathrm{mmol} \mathrm{1} \mathrm{H}^{-1}\right)$ \\
\hline Control & 0 & 3 & $7.38 \pm 0.05$ & $3.52 \pm 0.09$ & $13.38 \pm 0.05$ & $4.97 \pm 0.55$ \\
& 6 & 3 & $7.38 \pm 0.06$ & $3.53 \pm 0.07$ & $13.37 \pm 0.05$ & $5.40 \pm 0.60$ \\
LS & 0 & 3 & $7.40 \pm 0.05$ & $3.56 \pm 0.05$ & $13.38 \pm 0.07$ & $4.90 \pm 0.78$ \\
& 6 & 3 & $7.40 \pm 0.08$ & $3.54 \pm 0.06$ & $13.37 \pm 0.06$ & $4.77 \pm 0.61$ \\
MS & 0 & 3 & $7.36 \pm 0.06$ & $3.55 \pm 0.04$ & $13.38 \pm 0.05$ & $5.07 \pm 0.47$ \\
& 6 & 3 & $7.42 \pm 0.07$ & $3.57 \pm 0.08$ & $13.37 \pm 0.07$ & $5.37 \pm 0.59$ \\
HS & 0 & 3 & $7.39 \pm 0.05$ & $3.51 \pm 0.08$ & $13.40 \pm 0.04$ & $5.40 \pm 0.66$ \\
& 6 & 3 & $7.39 \pm 0.05$ & $3.59 \pm 0.07$ & $13.38 \pm 0.06$ & $5.30 \pm 0.46$ \\
\hline
\end{tabular}

Arterial blood $\mathrm{pH}, \mathrm{PaCO}_{2}, \mathrm{PaO}_{2}$ and glucose levels were not statistically different following sevoflurane exposure as analyzed by t-test, all $\mathrm{P}>0.05)$. $\mathrm{PaCO}_{2}$, partial pressure of carbon dioxide; $\mathrm{PaO}_{2}$, partial pressure of oxygen; LS, subanesthetic concentration, $0.1 \mathrm{MAC}$; MS, subclinical concentration, 0.3 MAC; HS, highest tolerated concentration, 0.7 MAC.

began $24-48 \mathrm{~h}$ after the last habituation period. For subsequent object location and object-in-place tests, tests began 24-48 h after one habituation period. The objects used in the tests were made of glass and were of a similar size $(10 \times 10 \times 10 \mathrm{~cm})$ but different shapes (Fig. 1). Novel objects were placed in a counterbalanced position to eliminate any side preference of the rats. Each test consisted of a sample and a test phase, and the objects were placed $10 \mathrm{~cm}$ away from the walls in the box. A rat was released into the box facing the wall. Between each rat release, the box was wiped again with $40 \%$ ethanol.

Object recognition. In the sample phase (4 min), two identical objects were placed for the subject rat to explore (Fig. 1A). Then, the subject was placed back into its colony cage. After $24 \mathrm{~h}$, the test phase for evaluating object memory began. In the test phase (4 min), one of the two previously identical objects was replaced by a novel object with a different shape. The subject was again released into the box for exploration.

Object location recognition. This test was performed one week after the object recognition test. In the sample phase (4 min), another two identical objects were placed for the subject rat to explore (Fig. 1C). Then, the subject was placed back into its colony cage. After $24 \mathrm{~h}$, the test phase for evaluating object location memory began. In the test phase $(4 \mathrm{~min})$, one of the two previously identical objects was moved to another corner of the box. The subject was then released into the box for exploration.

Object-in-place recognition. This test was performed one week after the object location test. In the sample phase (5 min), four different objects were placed for the subject rat to explore (Fig. 1E). Then, the subject was placed back into its colony cage. After $1 \mathrm{~h}$, the test phase for evaluating object-in-place memory began. In the test phase $(5 \mathrm{~min})$, the two previous objects were interchanged in position (Fig. 1E). The subject was then released into the box for exploration. The longer time spent investigating the pair of objects that switched positions, the stronger the object-in-place memory of the subject.

Recognition memory. One investigator blinded to the group allocation of the rats recorded the exploring time (21). The time when the subject rat directed its nose to explore an object within $2 \mathrm{~cm}$ was considered active exploring behavior. As previous studies have indicated, recognition memory is most sensitive in the first 1-2 min of the test phase (22-24), the discrimination ratio (DR) was calculated only from data obtained during the first $2 \mathrm{~min}$ of the test phase. DR was defined as the time the subject rat spent exploring the novel object(s) or position(s) divided by the total exploration time in the test phase. Rats that scored a higher DR exhibited stronger recognition memory and discrimination ability.

Statistical analysis. Statistical analysis was conducted using SPSS, version 20 for Windows (IBM SPSS, Armonk, NY, USA) and $\mathrm{P}<0.05$ was considered to indicate a statistically significant difference. The standard error of the mean is presented as error bars on all figures. The arterial blood gas analysis data ( $\mathrm{pH}, \mathrm{PaCO}_{2}, \mathrm{PaO}_{2}$ and glucose levels) prior to and following exposure to sevoflurane/air were compared using Student's t-test. The ELISA, TUNEL staining and behavioral studies data were analyzed using one-way analysis of variance (ANOVA). The Bonferroni method was employed for multiple comparisons among the four groups.

\section{Results}

Arterial blood gas following sevoflurane treatment. Arterial blood gas analyses (arterial blood $\mathrm{pH}, \mathrm{PaCO}_{2}, \mathrm{PaO}_{2}$ and glucose levels) prior to and following sevoflurane/air exposure were not significantly different as compared by Student's t-test (Table I), indicating the absence of severe apnea and hypoxemia during anesthesia.

Antioxidant levels following sevoflurane treatment. No significant difference to the levels of SOD were identified between each group. SOD concentrations in the plasma of the control, LS, MS and HS groups were 8.37 $\pm 0.42,8.55 \pm 0.38,8.40 \pm 0.42$ and $8.42 \pm 0.39 \mathrm{U} / \mathrm{ml}$, respectively $(\mathrm{F}=0.471, \mathrm{P}=0.704)$. SOD concentrations in the hippocampal homogenate of the control, LS, MS and HS groups were 11.11 $\pm 1.47,10.67 \pm 1.45,11.57 \pm 1.33$ and $12.43 \pm 1.83 \mathrm{U} / \mathrm{ml}$, respectively $(\mathrm{F}=2.907, \mathrm{P}=0.045)$. Plasma 
Table II. Total exploration time ( $\mathrm{sec}$, mean \pm standard deviation) in the sample phase and test phase.

\begin{tabular}{|c|c|c|c|c|c|c|}
\hline \multirow[b]{2}{*}{ Treatment } & \multicolumn{2}{|c|}{ Object recognition } & \multicolumn{2}{|c|}{ Object location } & \multicolumn{2}{|c|}{ Object-in-place } \\
\hline & Sample phase & Test phase & Sample phase & Test phase & Sample phase & Test phase \\
\hline Control & $21.0 \pm 2.2$ & $24.0 \pm 3.3$ & $20.0 \pm 9.9$ & $51.8 \pm 8.3$ & $21.0 \pm 2.2$ & $24.0 \pm 3.3$ \\
\hline LS & $21.8 \pm 4.3$ & $20.7 \pm 3.6$ & $18.7 \pm 13.0$ & $50.0 \pm 9.5$ & $21.8 \pm 4.3$ & $20.7 \pm 3.6$ \\
\hline MS & $22.8 \pm 3.9$ & $21.8 \pm 10.3$ & $35.0 \pm 4.4$ & $20.2 \pm 5.9$ & $22.8 \pm 3.9$ & $21.8 \pm 10.3$ \\
\hline HS & $26.2 \pm 1.9$ & $23.2 \pm 1.9$ & $55.3 \pm 7.7$ & $37.5 \pm 7.1$ & $26.2 \pm 1.9$ & $23.2 \pm 1.9$ \\
\hline
\end{tabular}

Data show the total exploration time (sec) and are presented as the mean \pm standard deviation. LS, subanesthetic concentration, 0.1 MAC; MS, subclinical concentration, 0.3 MAC; HS, highest tolerated concentration, 0.7 MAC.

A

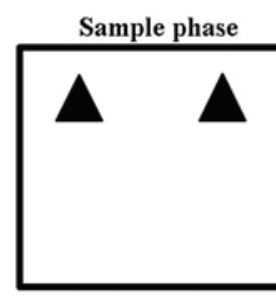

$\mathbf{C}$

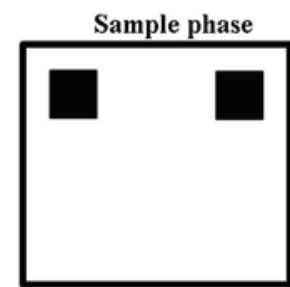

$\mathbf{E}$

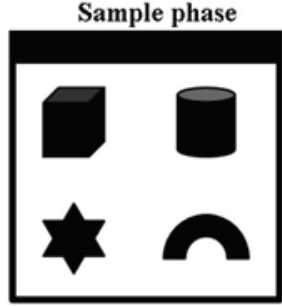

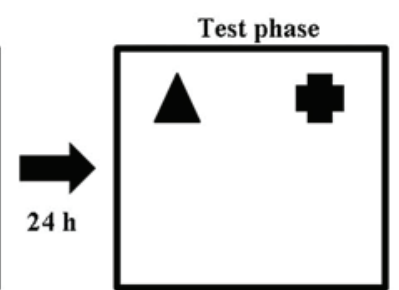

Test phase

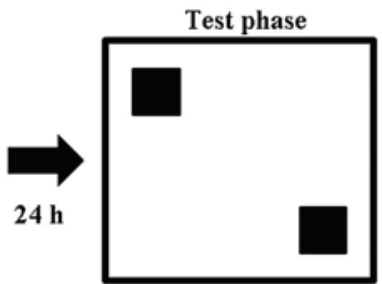

Test phase

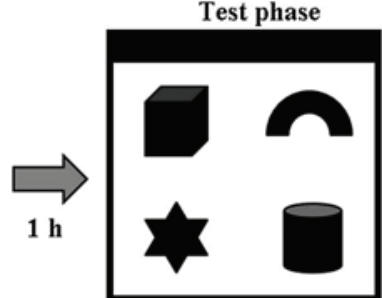

B
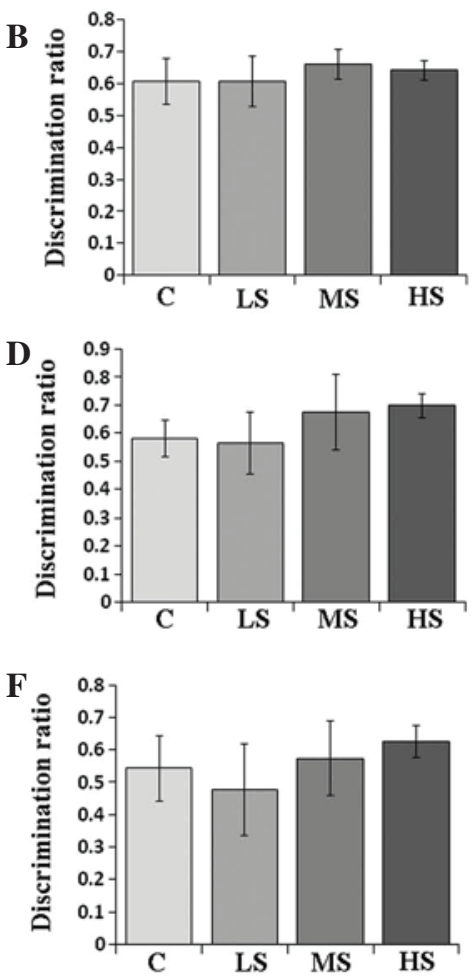

Figure 1. Demonstration of the object exploration tests and test results in the air control and sevoflurane-treated groups. (A) Diagram and (B) results of the object recognition memory test in the air control and sevoflurane-treated groups. (C) Diagram and (D) results of the object location recognition memory test in the air control and sevoflurane-treated groups. (E) Diagram and (F) results of the object-in-place recognition memory test in the air control and sevoflurane-treated groups. No significant difference was indicated among the groups. All results are presented as the mean \pm standard deviation. LS, subanesthetic concentration, 0.1 MAC; MS, subclinical concentration, 0.3 MAC; HS, highest tolerated concentration, 0.7 MAC.

and hippocampal GSH-px levels decreased when the concentration was $>1.3 \%$. GSH-px concentrations in the plasma of the control, LS, MS and HS groups were 4.66 $\pm 0.38,4.53 \pm 0.38$, $4.07 \pm 0.26$ and $4.25 \pm 0.22 \mathrm{U} / 1$, respectively $(\mathrm{F}=8.492, \mathrm{P}<0.001)$. GSH-px concentrations in the hippocampal homogenate of the control, LS, MS and HS groups were 7.75 $\pm 0.72,7.02 \pm 0.50$, $5.33 \pm 0.44$ and $5.87 \pm 0.66 \mathrm{U} / 1$, respectively $(\mathrm{F}=40.880$, $\mathrm{P}<0.001)$. MDA levels increased following treatment with sevoflurane, however, a paradoxical decrease of MDA was observed when a subclinical concentration was administered (Fig. 2). MDA concentrations in the plasma of the control, LS, MS and HS groups were $0.71 \pm 0.05,0.87 \pm 0.07,0.71 \pm 0.04$ and $0.93 \pm 0.09 \mathrm{nmol} / \mathrm{ml}$, respectively $(\mathrm{F}=37.226, \mathrm{P}<0.001)$. MDA concentrations in the hippocampal homogenate of the control,
LS, MS and HS groups were 1.14 $\pm 0.14,1.65 \pm 0.18,1.21 \pm 0.20$ and $1.71 \pm 0.20 \mathrm{nmol} / \mathrm{ml}$, respectively $(\mathrm{F}=31.974, \mathrm{P}<0.001)$.

Neuronalapoptosisfollowing sevofluranetreatment. Sevoflurane induced hippocampal neuronal apoptosis in a concentration-dependent manner. The number of TUNEL-positive neurons increased as the concentration of sevoflurane increased (Fig. 3). The number of TUNEL positive neurons in the control, LS, MS and HS groups were 17.4 $\pm 5.0,26.8 \pm 5.76,39.5 \pm 6.4$ and 54.0 \pm 6.7 cells $/ 100 \mu \mathrm{m}^{2} \mathrm{CA} 3$ area, respectively $(\mathrm{F}=14.069$, $\mathrm{P}<0.01)$

Total exploration times in the behavioural studies. Total exploration times in the object recognition test are listed in 
A

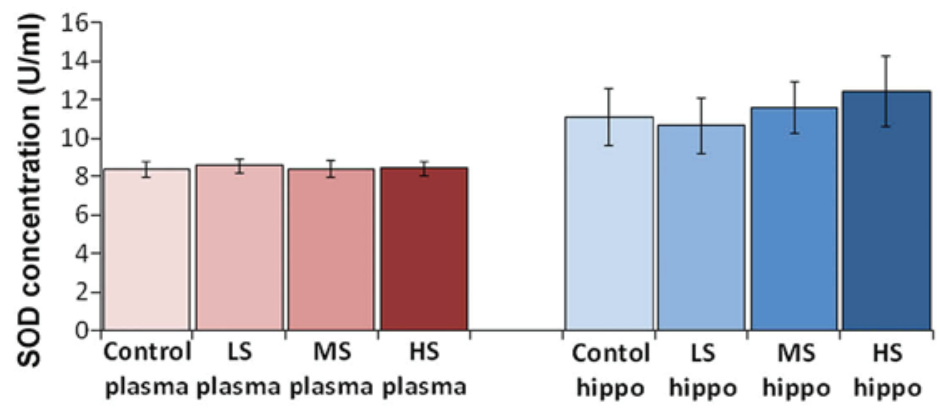

B
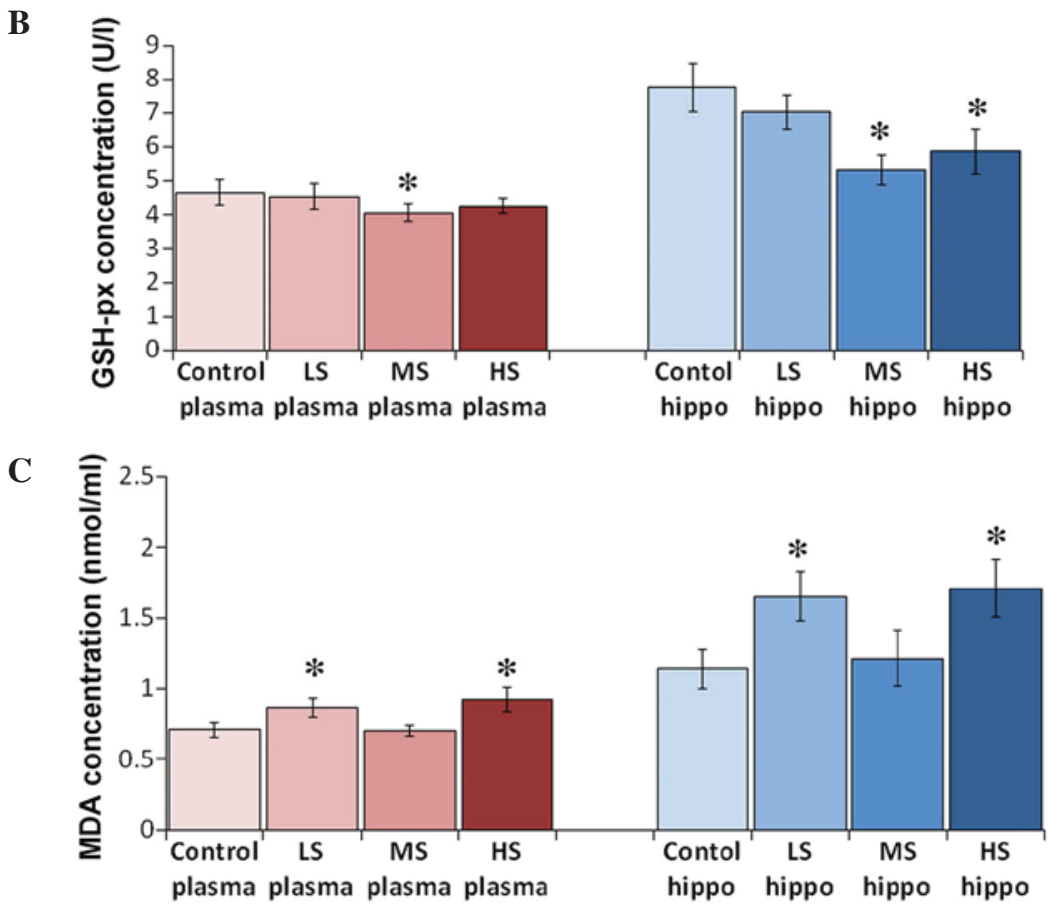

Figure 2. Levels of SOD, GSH-px, and MDA in plasma and hippocampus by ELISA. Levels of (A) SOD, (B) GSH-px and (C) MDA in the plasma and the hippocampus by ELISA. All results are presented as the mean \pm standard deviation, ${ }^{*} \mathrm{P}<0.01$ vs. the control group. LS, subanesthetic concentration, $0.1 \mathrm{MAC}$; MS, subclinical concentration, 0.3 MAC; HS, highest tolerated concentration, 0.7 MAC; SOD, superoxide dismutase; GSH-px, glutathione peroxidase; MDA, malondialdehyde; ELISA, enzyme-linked immunosorbent assay.

Table II. The discrimination ratios of the control, LS, MS and HS groups in the object recognition test were $0.608 \pm 0.071$, $0.607 \pm 0.080,0.662 \pm 0.047,0.642 \pm 0.031$, respectively. The discrimination ratio was analyzed by one-way ANOVA, and the result indicated no significant difference among the groups ( $F=0.978, P=0.423$; Fig. 1). Treatment with different concentrations of sevoflurane did not influence the discrimination ability that depends on object recognition memory.

Total exploration times in the object location recognition test are also listed in Table II. The discrimination ratios of control, LS, MS and HS groups in the object location recognition test were $0.582 \pm 0.066,0.565 \pm 0.109,0.677 \pm 0.134$ and $0.698 \pm 0.042$, respectively. The discrimination ratio was analyzed with one-way ANOVA, and the result demonstrated no significant difference among the groups $(\mathrm{F}=2.485, \mathrm{P}=0.090$; Fig. 1). Treatment with different concentrations of sevoflurane did not influence discrimination ability that depends on object location recognition memory.

Total exploration times in the object-in-place recognition test are listed in Table II. The discrimination ratio of control, LS, MS and HS groups in the object-in-place recognition test were $0.543 \pm 0.100,0.477 \pm 0.142,0.573 \pm 0.115$ and $0.625 \pm 0.051$, respectively. The discrimination ratio was analyzed with one-way ANOVA, and the result demonstrated no significant difference among the groups $(\mathrm{F}=1.670, \mathrm{P}=0.205$; Fig. 1). Treatment with different concentrations of sevoflurane did not influence the discrimination ability that depends on object-in-place recognition memory.

\section{Discussion}

SOD catalyzes the dismutation of superoxide into oxygen and hydrogen peroxide, thereby serving as an essential antioxidant in the body. It exerts anti-inflammatory effects and protects cells from damage. GSH-px is another enzyme that serves to reduce oxidative damage and protect the structural integrity and function of cell membranes (25). SOD and GSH-px are major antioxidant enzymes that eliminate free radicals and have marked anti-oxidative stress effects, maintaining an equilibrium between oxidants and antioxidants in the body (26). By contrast, MDA, the direct product of lipid peroxidation, serves as an indicator of the extent of cell damage. MDA may disrupt 
A

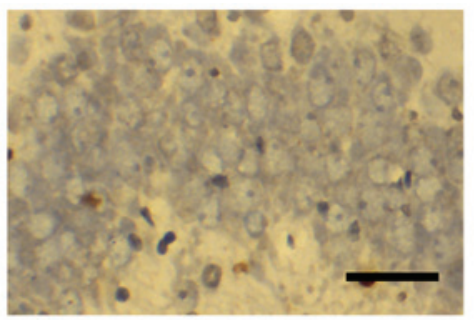

C

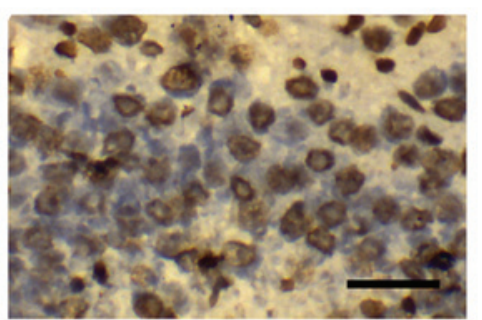

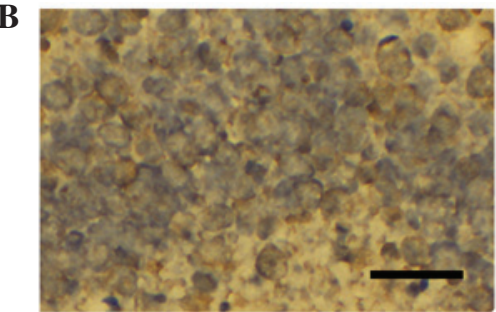

D

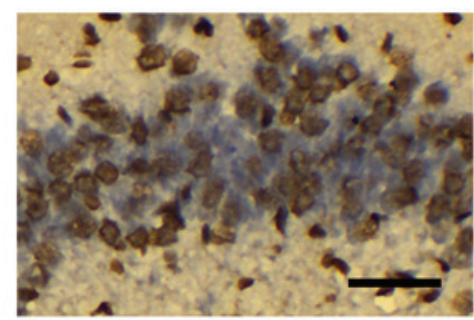

$\mathbf{E}$

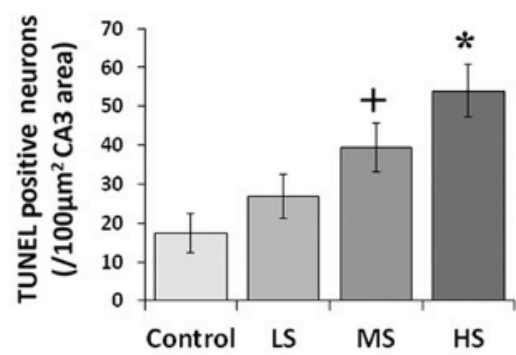

Figure 3. TUNEL staining in the hippocampus (CA3) and TUNEL-positive neuron count. Representative photomicrographs of TUNEL staining of coronal sections of the hippocampus of (A) air control post-natal day 7 rats and rats treated with (B) LS, (C) MS and (D) HS). Scale bar, $50 \mu \mathrm{m}$. (E) Comparison of TUNEL-positive neuron numbers between the air control and sevoflurane-treated groups. All data are presented as the mean \pm standard deviation. ${ }^{+} \mathrm{P}<0.05$ and ${ }^{*} \mathrm{P}<0.01$ vs. the control group. CA3, region III of hippocampus proper; LS, subanesthetic concentration, $0.1 \mathrm{MAC}$; MS, subclinical concentration, $0.3 \mathrm{MAC}$; HS, highest tolerated concentration, 0.7 MAC; TUNEL, terminal deoxynucleotidyl-transferase dUTP nick end labeling.

cell membrane structure, lead to DNA fragmentation and hasten apoptosis (27). Therefore, determination of MDA, SOD and GSH-px levels in the plasma and the hippocampus indicate the general state of free radical metabolism in the body and the extent of hippocampal damage during sevoflurane anesthesia.

Free radicals destroy the structure of the cell membrane structure and attack DNA, fracturing it and increasing the rate of apoptosis. They are considered to be closely correlated with inflammatory processes and tumorigenesis (28). As TUNEL staining detects DNA fragmentation in apoptotic cells (29), the increased production of MDA demonstrated in the plasma and the hippocampus possibly accelerates the process of apoptosis in the sevoflurane-treated animals.

Mammalian cells require an appropriate balance of oxidants and antioxidants (10). The elevation of MDA in the plasma of sevoflurane-treated rats suggests that a large number of oxygen free radicals accumulate in the body, and an imbalance occurs between free radical production and detoxification. Such an imbalance decreases the activity of SOD and GSH-px and increases the levels of MDA, driving the anti-oxidation system into a low energy state. More lipid peroxidation occurs than the antioxidant defense systems process, thus generating MDA, the end product of lipid peroxidation.

The relatively low level of plasma and hippocampal MDA that paradoxically appeared in the MS group suggests that sevoflurane may reduce oxidative stress at the subclinical concentration of 0.3 MAC (1.3\%). However, this benign effect did not appear to ameliorate the apoptotic process in the hippocampus, indicating that an unknown pathway rather than the oxidative system affects sevoflurane-induced apoptosis.

Sevoflurane was demonstrated to impact the antioxidative status of erythrocytes characterized by decreased SOD and increased GSH-px (30). The preservation of erythrocytes was observed to be compromised in surgery with sevoflurane instead of propofol anesthesia, which indicates that sevoflurane may result in oxidative stress (31). By contrast, Allaouchiche et al (32) investigated the oxidative status of the circulation and lungs during sevoflurane anesthesia at $1 \mathrm{MAC}$ and observed relatively low levels of MDA and GSH-px in the plasma and bronchoalveolar lavage fluid compared with propofol and desflurane anesthesia, indicating that antioxidant properties of sevoflurane may exist. Similarly, Turkan et al (33) investigated oxidative stress in the liver, brain, kidney and lung tissue of rats exposed to $8 \%$ sevoflurane for $4 \mathrm{~h}$ and observed MDA to be significantly decreased. However, in the majority of clinical scenarios, sevoflurane is co-administered with narcotics, such as an opioid, during surgery, thus a concentration $>1$ MAC is rarely employed for maintenance of anesthesia (34).

These findings suggest that the proper application of sevoflurane at a subclinical concentration may lower general oxidative stress levels in the body.

Novel object recognition tasks are a method of assessing recognition memory in rodents (35). The hippocampus is involved in recognition memory when such memory involves remembering a particular stimulus that occurred in a particular place or when the memory contains a temporal or 
object proximity component (36). Lesions to the hippocampus produce moderate and reliable recognition memory impairments (35). Despite the fact that sevoflurane reliably induces neuronal apoptosis in the postnatal brain, no long-term impact on recognition memory was observed in the present study.

In conclusion, although early exposure to a subclinical concentration of sevoflurane reduces oxidative stress, it does not prevent the process of sevoflurane-induced hippocampal apoptosis. These changes do not affect subsequent recognition memory in juvenile rats. The current study suggested collection of human data regarding anti-oxidation measures and their clinical outcome is required, particularly for the developing brain.

\section{Acknowledgements}

This study was supported by funding from the B. Braun Research Fund for Anesthesiology (grant no. BBDF-2014-016); the Guangdong Science and Technology Planning Project (grant no. 2013B051000045); and the Guangzhou International Science and Technology Cooperation Project (grant no. 2012J5100019). The sources of funding had no role in the design and conduct of this project or in the preparation of the manuscript.

\section{References}

1. Mellon RD, Simone AF and Rappaport BA: Use of anesthetic agents in neonates and young children. Anesth Analg 104: 509-520, 2007.

2. Sarner JB, Levine M, Davis PJ, Lerman J, Cook DR and Motoyama EK: Clinical characteristics of sevoflurane in children. A comparison with halothane. Anesthesiology 82: 38-46, 1995.

3. Payne RS, Akca O, Roewer N, Schurr A and Kehl F: Sevoflurane-induced preconditioning protects against cerebra ischemic neuronal damage in rats. Brain Res 1034: 147-152, 2005.

4. Zuo Z: A novel mechanism for sevoflurane preconditioning-induced neuroprotection. Anesthesiology 117: 942-944, 2012.

5. Zhang X, Xue Z and Sun A: Subclinical concentration of sevoflurane potentiates neuronal apoptosis in the developing C57BL/6 mouse brain. Neuroscience letters 447: 109-114, 2008.

6. Satomoto M, Satoh Y, Terui K, Miyao H, Takishima K, Ito M and Imaki J: Neonatal exposure to sevoflurane induces abnormal social behaviors and deficits in fear conditioning in mice. Anesthesiology 110: 628-637, 2009.

7. Komatsu H, Ohara T, Nogaya J, Yokono S and Ogli K: Subanesthetic concentrations of volatile anesthetics may enhance acquired avoidance training in ddN mice. Anesth Analg 73: 295-299, 1991.

8. Komatsu H, Nogaya J, Kuratani N, Ueki M, Yokono S and Ogli K : Psychomotor performance during initial stage of exposure to halothane, enflurane, isoflurane and sevoflurane in mice. Clin Exp Pharmacol Physiol 24: 706-709, 1997.

9. Otsubo T, Maekawa M, Nagai T, Sakio H and Hori Y: Facilitatory effects of subanesthetic sevoflurane on excitatory synaptic transmission and synaptic plasticity in the mouse hippocampal CA1 area. Brain Res 1197: 32-39, 2008.

10. Buttke TM and Sandstrom PA: Oxidative stress as a mediator of apoptosis. Immunol Today 15: 7-10, 1994.

11. Orliaguet G, Vivien B, Langeron O, Bouhemad B, Coriat P and Riou B: Minimum alveolar concentration of volatile anesthetics in rats during postnatal maturation. Anesthesiology 95: 734-739, 2001

12. Alkire MT, Nathan SV and McReynolds JR: Memory enhancing effect of low-dose sevoflurane does not occur in basolateral amygdala-lesioned rats. Anesthesiology 103: 1167-1173, 2005.

13. Alkire MT and Gorski LA: Relative amnesic potency of five inhalational anesthetics follows the Meyer-Overton rule. Anesthesiology 101: 417-429, 2004.

14. Feng X, Liu JJ,Zhou X, Song FH, Yang XY, Chen XS, Huang WQ, Zhou LH and Ye JH: Single sevoflurane exposure decreases neuronal nitric oxide synthase levels in the hippocampus of developing rats. Br J Anaesth 109: 225-233, 2012.
15. Zhou X, Song FH, He W, Yang XY, Zhou ZB, Feng X and Zhou LH: Neonatal exposure to sevoflurane causes apoptosis and reduces nNOS protein expression in rat hippocampus. Mol Med Rep 6: 543-546, 2012.

16. Young C, Jevtovic-Todorovic V, Qin YQ, Tenkova T, Wang H, Labruyere J and Olney JW: Potential of ketamine and midazolam, individually or in combination, to induce apoptotic neurodegeneration in the infant mouse brain. Br J Pharmacol 146: 189-197, 2005.

17. Lu LX, Yon JH, Carter LB and Jevtovic-Todorovic V: General anesthesia activates BDNF-dependent neuroapoptosis in the developing rat brain. Apoptosis 11: 1603-1615, 2006.

18. Wang J, Yan L, Zhao X, Wu W and Zhou LH: The diversity of nNOS gene expression in avulsion-injured spinal motoneurons among laboratory rodents. Nitric Oxide 22: 37-42, 2010.

19. Ananth C, Dheen ST, Gopalakrishnakone P and Kaur C: Distribution of NADPH-diaphorase and expression of nNOS, $\mathrm{N}$-methyl-D-aspartate receptor (NMDAR1) and non-NMDA glutamate receptor (GlutR2) genes in the neurons of the hippocampus after domoic acid-induced lesions in adult rats. Hippocampus 13: 260-272, 2003.

20. Howland JG, Cazakoff BN and Zhang Y: Altered object-in-place recognition memory, prepulse inhibition and locomotor activity in the offspring of rats exposed to a viral mimetic during pregnancy. Neuroscience 201: 184-198, 2012.

21. Howland JG and Cazakoff BN: Effects of acute stress and GluN2B-containing NMDA receptor antagonism on object and object-place recognition memory. Neurobiol Learn Mem 93: 261-267, 2010.

22. Dix SL and Aggleton JP: Extending the spontaneous preference test of recognition: Evidence of object-location and object-context recognition. Behav Brain Res 99: 191-200, 1999.

23. Clark RE, Zola SM and Squire LR: Impaired recognition memory in rats after damage to the hippocampus. J Neurosci 20: 8853-8860, 2000.

24. Barker GR and Warburton EC: NMDA receptor plasticity in the perirhinal and prefrontal cortices is crucial for the acquisition of long-term object-in-place associative memory. J Neurosci 28: 2837-2844, 2008.

25. Drabko K, Bojarska-Junak A and Kowalczyk J: Activity of superoxide dismutase and glutathione peroxidase and concentrations of malonyldialdehyde, vitamin $\mathrm{E}$, total antioxidant status and extracellular cytokines concentrations in children with acute lymphoblastic leukaemia (ALL). Med Wieku Rozwoj 10: 861-868, 2006 (In Polish).

26. Aguilar A, Alvarez-Vijande R, Capdevila S, Alcoberro J and Alcaraz A: Antioxidant patterns (superoxide dismutase, glutathione reductase, and glutathione peroxidase) in kidneys from non-heart-beating-donors: Experimental study. Transplant Proc 39: 249-252, 2007.

27. Surapaneni KM and Venkataramana G: Status of lipid peroxidation, glutathione, ascorbic acid, vitamin $\mathrm{E}$ and antioxidant enzymes in patients with osteoarthritis. Indian J Med Sci 61: 9-14, 2007.

28. Djordjevic VB: Free radicals in cell biology. Int Rev Cytol 237: 57-89, 2004.

29. Gavrieli Y, Sherman Y and Ben-Sasson SA: Identification of programmed cell death in situ via specific labeling of nuclear DNA fragmentation. J Cell Biol 119: 493-501, 1992.

30. Turkan H, Aydin A, Sayal A and Karahalil B: The effect of sevoflurane and desflurane on markers of oxidative status in erythrocyte. Toxicol Ind Health 27: 181-186, 2011.

31. Tsuchiya M, Asada A, Kasahara E, Sato EF, Shindo M and Inoue M: Antioxidant protection of propofol and its recycling in erythrocyte membranes. Am J Respir Crit Care Med 165: 54-60, 2002.

32. Allaouchiche B, Debon R, Goudable J, Chassard D and Duflo F: Oxidative stress status during exposure to propofol, sevoflurane and desflurane. Anesth Analg 93: 981-985, 2001.

33. Turkan H, Aydin A, Sayal A, Eken A, Akay C and Karahalil B: Oxidative and antioxidative effects of desflurane and sevoflurane on rat tissue in vivo. Arh Hig Rada Toksikol 62: 113-119, 2011.

34. Wang HL, Yang L, Guo XY, Zhang LP, Bi SS and Lu W: Response surface analysis of sevoflurane-remifentanil interactions on consciousness during anesthesia. Chinese Med J (Engl) 125: 2682-2687, 2012.

35. Broadbent NJ, Gaskin S, Squire LR and Clark RE: Object recognition memory and the rodent hippocampus. Learn Mem 17: 5-11, 2009.

36. Barker GR and Warburton EC: When is the hippocampus involved in recognition memory? J Neurosci 31: 10721-10731, 2011. 